\title{
Vortices in micro/nano channel flows
}

\author{
Z.-H. Silber-Li ${ }^{1}$, X. Zheng ${ }^{1}$, G.-P. Kong ${ }^{1} \&$ T. H. Moulden ${ }^{2}$ \\ ${ }^{1}$ Chinese Academy of Sciences, China \\ ${ }^{2}$ The University of Tennessee Space Institute, USA
}

\begin{abstract}
The paper describes a series of experiments that study the formation of threedimensional vortices in micro/nano channel flows subject to an applied electric field. The discussion is supported by a theoretical discussion of the vorticity generation process in such flows.

Keywords: micro/nano flows, vorticity, electric fields.

“......

Under Maple Bridge the boat is moored

For my long journey will resume at dawn"

Chang Chi ( $8^{\text {th }}$ century).
\end{abstract}

\section{Introduction}

A good reason exists as to why electro-osmosis flows are coming into common use at the micro/nano scale. For small diameter fluidic devices the flow rate, $Q$, is given as $Q=\pi D^{4} P /(128 \mu L)$ and the Reynolds number low. Hence for pressure driven flows operating a fixed flow rate, the pressure required varies as $D^{-4}$ and becomes very large as $D \rightarrow 0$. On the other hand, for electro-osmotic driven flows, there is only a $D^{-2}$ proportionality.

For electro-osmotic flows in simple channels, the flow is uniform outside a thin, viscous, charge layer that exists near the channel walls. This, of course, is no longer true if the channel contains large area changes when flow separation could occur. The study of Takhistov et al. [1] demonstrated, both theoretically and experimentally, that electrolyte depletion occurs at the junction between a wide $(400 \mu \mathrm{m})$ diameter and a small $(80 \mu \mathrm{m})$ diameter channel. Vortical flow was also observed in that flow and, it was suggested, changes to the local zeta potential gave 
rise to the vortex motion. Park et al. [2] provided a small disturbance analysis of the electro-osmotic flow in a bottleneck geometry and found that both vortical flow and an EOF profile distortion occurred in the constriction. These authors postulated that the presence of eddies was partly geometric, the flow passing into a narrow constriction, and partly due to a redistribution of ions. Kim et al. [3] first observed vortices located at the corners of a micro/nano channel using $500 \mathrm{~nm}$ fluorescent particles and drew attention to the importance of concentration polarization initiated near the nano-channel. They located two pairs of vortices near the sidewalls of the micro-channel which were due, it was suggested, to the non-equilibrium electro-osmotic flow. Huang and Yang [4] visualized the recirculating flow field using $2 \mu \mathrm{m}$ latex particles and simulated the vortices inside the channel during their study of the depletion and enrichment process in a micro/nano-channel. Hybrid micro/nano channels have also been constructed from two separate micro-channels separated by a porous membrane. For example, Jin et al. [5] simulated the flow field in this kind device and noticed electro-osmotic flow of the second kind. These authors suggested that the net space charge in the depletion region gave rise to non-linear electrokinetic transport (the second kind electro-osmotic flow) and hence to vortical motion.

Two distinct flow mechanisms are at work in these devices:

1) the electro-osmotic generation of vorticity as described in section 2

2) discrete vortical flow development

Some vortex flows (such as those produced in sudden enlargement of a channel where wall vorticity has been generated) are the same in electro-osmotic flows as in familiar fluid dynamic flows; but with the presence of additional vorticity from the electric field (as in equation (6) below). The underlying fluid dynamics cannot be ignored. For example, the usual viscous vorticity creation mechanism is still present and need not be discussed herein. Note that Moffatt [6] computed corner eddies in low Reynolds number flow.

Micro/nano channel devices have potential application in many important practical situations. Hence the operational properties, and the physics associated with that operation, must be understood. In particular, does any vortical motion found in such flows enhance, or detract from, the intended operation of the device? The objective in the present paper is to observe the vortices in a three-dimensional hybrid micro/nanochannel using fluorescent particles of $200 \mathrm{~nm}$ diameter and to establish the theoretical structure required to explore the electro-osmotic vorticity creation mechanism. This requires a study of both the Navier Stokes and NernstPlanck equations.

\section{Theoretical background}

It is assumed for the theoretical structure discussed below that the fluid could be treated as a continuum. Thus, the smallest length scale associated with the experiment described in section 3 was that of the pores, of the order of $50 \mathrm{~nm}$, in the nano-porous membrane. For the fluids of interest in the experiments reported below (conducted at room temperature) there were approximately $10^{10}$ molecules 
per $(\mu m)^{3}$ for both DI water and the buffer solution. This implied a pore-Knudsen number of $K_{n} \approx 0.04$.

\subsection{Some theorems}

Given this finding, it is appropriate to adopt the Navier Stokes equations as a model of the fluid motion inside a micro/nano scale device. Since the fluid velocities are very low with respect to the sound speed, it is also appropriate to assume that the fluid density is a constant. With these assumptions, the field equations for the fluid motion are given as:

$$
\frac{\partial v_{i}}{\partial x_{i}}=0 ; \quad \frac{\partial v_{i}}{\partial t}+v_{j} \frac{\partial v_{i}}{\partial x_{j}}+\frac{\partial P}{\partial x_{i}}+\nu \frac{\partial^{2} v_{i}}{\partial x_{j} \partial x_{j}}+f_{i}
$$

In equation (1b), $P(\mathbf{x}, t)$ denotes the fluid pressure normalized by the fluid density and $\mathbf{v}(\mathbf{x}, t)$ represents the fluid velocity. $\nu$ is the, assumed constant, kinematic viscosity of the fluid. $\mathbf{f}(\mathbf{x}, t)$ represents a body force which in the present application will be Lorentz (see equation $2 \mathrm{a}$ ).

Gurtin [7] gave a simple uniqueness theorem in the form:

Result: Let $\left(\mathbf{v}_{1}, P_{1}\right)$ and $\left(\mathbf{v}_{2}, P_{2}\right)$ be two solutions of the equations $(1 a, b)$ (with the same boundary conditions and an independent body force). Then: $\mathbf{v}_{1}=\mathbf{v}_{2} ; \quad P_{1}=P_{2}+Q(t) ; \quad$ such that the quantity $Q=Q(t)$ is an arbitrary function of time.

and is valid provided that the regularity required by the proof holds. This regularity concerns the existence of a finite velocity gradient tensor $\mathbf{L}$; that is $\|\mathbf{L}\| \equiv$ $\|\partial \mathbf{v} / \partial \mathbf{x}\|<\infty$. The result of Gurtin [7] shows that while the velocity field is specified uniquely by the field equations, the pressure field need not be unique. This independence of the pressure level has been known experimentally since the work of Dubuat (a fact noted by Stokes [8]).

While the above result of Gurtin [7] is of importance for many applications, it is not appropriate in the present circumstances since the Lorentz body force, is dependent upon the flow variables. Specifically:

$$
\mathbf{f}=\rho_{e} \mathbf{E} \quad \text { with } \quad \rho_{e}=F \sum\left(z^{\alpha} c^{\alpha}\right)
$$

Here $\rho_{e}$ denotes the charge density (with $F$ the Faraday constant and $z^{\alpha}$ the valence of charge $c^{\alpha}$ ) and $\mathbf{E}$ is the electric field. Now the charges, $c^{\alpha}$ are coupled with the fluid velocity, $\mathbf{v}(\mathbf{x}, t)$ in equations $(1 \mathrm{a}, \mathrm{b})$, via the Nernst-Planck flux and the charge conservation equation:

$\frac{\partial c^{\alpha}}{\partial t}+\frac{\partial J_{i}(\alpha)}{\partial x_{i}}=0 \quad$ with $\quad J_{i}(\alpha)=-D \frac{\partial c^{\alpha}}{\partial x_{i}}+v_{i} c^{\alpha}-\mu_{e p} z^{\alpha} F c^{\alpha} \frac{\partial \Psi}{\partial x_{i}}$

which completes the specification of the fluid motion. 
It is assumed that there are no magnetic fields, either applied or induced, acting on the fluid so that the Maxwell equations state that:

$$
\nabla^{2}(\Psi)=-\rho_{e} / \epsilon
$$

for the permittivity $\epsilon$ and electric potential, $\Psi$, defined such that the electric field $\mathbf{E}$ is given by $\mathbf{E}=-\nabla(\Psi)$. The Poisson equation (4) shows, directly, that if the charge density $\rho_{e}$ is uniquely defined then the potential $\Psi$ is unique and so is the electric field E. Uniqueness of the Poisson equation is discussed in Stakgold [9]. Vorticity generation by this process is discussed in more detail in Silber-Li et al. [10]. How this vorticity is wrapped into a discrete vortex depends upon the geometry of the device and cannot be established from the present equations in isolation.

The above system of equations can now be stated in the standard form:

$$
\begin{aligned}
\frac{\partial v_{i}}{\partial t}+v_{j} \frac{\partial v_{i}}{\partial x_{j}}+\frac{\partial P}{\partial x_{i}} & =\nu \frac{\partial^{2} v_{i}}{\partial x_{j} \partial x_{j}}+\rho_{e} E_{i} \\
\frac{\partial v_{i}}{\partial x_{i}} & =0 \\
\frac{\partial \rho_{e}}{\partial t}+v_{i} \frac{\partial \rho_{e}}{\partial x_{i}} & =D \frac{\partial \rho_{e}}{\partial x_{i} \partial x_{i}}-\mu_{e p} F z^{1}\left[\frac{\partial \rho_{e}}{\partial x_{i}} E_{i}+\rho_{e} \frac{\partial E_{i}}{\partial x_{i}}\right] \\
\frac{\partial E_{i}}{\partial x_{i}} & =\frac{\rho_{e}}{\epsilon}
\end{aligned}
$$

if it is assumed that there are the same number of positive and negative ions. Here $D$ denotes the diffusivity and $\mu_{e p}$ the mobility. Both are assumed to be constant in the following deliberations. The electric field provides directionality in equation (5a).

A result of fundamental physics can be noted from direct inspection:

Lemma I: The system of equations $(5 a, b, c, d)$ is not covariant under time reversal. Neither is it covariant under electric field reversal.

The first part of the lemma, lack of time reversibility, just recognizes that the processes involved are not "ideal" and some increase in entropy must take place as the flow field develops. The second part of the Lemma is more interesting and suggests, in the context of the experimental results to be discussed below, that reversal of the electric field cannot "unwind" the vorticity after it has started to develop.

With the assumptions made above, a simple estimate suggests that:

Lemma II: Let $\left(\mathbf{v}_{1}, P_{1}, \rho_{e}^{1}, \mathbf{E}_{1}\right)$ and $\left(\mathbf{v}_{2}, P_{2}, \rho_{e}^{2}, \mathbf{E}_{2}\right)$ be two solutions of the equations ( $5 a, b, c, d)$ (with the same boundary conditions). Then:

$$
\mathbf{v}_{1}=\mathbf{v}_{2} ; \quad P_{1}=P_{2}+Q(t) ; \quad \mathbf{E}_{1}=\mathbf{E}_{2} ; \quad \rho_{e}^{1}=\rho_{e}^{2}
$$

such that the quantity $Q=Q(t)$ is an arbitrary function of time. The physical constants of the fluid are assumed the same for both flows. 
and the experimentally observed repeatability of the physics of micro/nano scale flows would be contained in the flow model discussed above.

\subsection{Vorticity and pressure}

Let attention pass to the consideration of vorticity dynamics in micro/nano scale flows driven by a Lorentz force. The curl operator applied to equation (1b), with the Lorentz body force, gives the vorticity evolution equation:

$$
\frac{d \zeta_{i}}{d t}=\frac{\partial v_{i}}{\partial x_{j}} \zeta_{j}+\nu \nabla^{2} \zeta_{i}-F \epsilon_{i j k}\left[\sum z^{\alpha} \frac{\partial c^{\alpha}}{\partial x_{j}}\right] \frac{\partial \Psi}{\partial x_{k}}
$$

which differs from the classical form in fluid dynamics due to the specific nature of the Lorentz body force. Now it is clear that vorticity is generated by the cross product of the concentration gradient with the electric field gradient. Form an equation for the global norm, $|\boldsymbol{\zeta}|_{g}^{2}=\int_{D}\langle\boldsymbol{\zeta}, \boldsymbol{\zeta}\rangle d V$, of the vorticity vector as an integral over the entire device using equation (6). As discussed in Silber-Li et al. [11] the vorticity in the device establishes a global norm which evolves as:

$$
\frac{\partial}{\partial t}|\zeta|_{g}^{2}+\nu L_{\zeta}^{2}|\zeta|_{g}^{2} \leq 2\|\mathbf{L}\|_{g}|\zeta|_{g}^{2}+\frac{1}{\nu L_{\zeta}^{2}}|\mathbf{g}|_{g}^{2}
$$

which couples to the velocity field via the velocity gradient $\mathbf{L}=\nabla(\mathbf{v})$ : the magnitude of the velocity global norm influences the rate of increase on global vorticity. More important is the influence of the vector $\mathbf{g}=\operatorname{curl}(\mathbf{f})$ which, for the present application, is bounded in norm by:

$$
|\mathbf{g}|_{g}^{2} \leq 2|\mathbf{E}|_{g}^{2}\left|\nabla\left(\rho_{e}\right)\right|_{g}^{2}
$$

to give an upper bound on the electro-osmotic vorticity generation process.

For reference note that the divergence operation applied to equation (1b) generates a Poisson equation for the fluid pressure which has the form:

$$
\nabla^{2} P=\frac{1}{\rho}\left[\frac{\partial \rho_{e}}{\partial x_{i}} E_{i}+\rho_{e} \frac{\partial E_{i}}{\partial x_{i}}\right]-\frac{\partial v_{j}}{\partial x_{i}} \frac{\partial v_{i}}{\partial x_{j}}
$$

Both the hydrodynamics and the electric field establish the pressure.

While the concepts leading to equations (6) and (7) are quite standard in classical fluid mechanics, their implications for micro/nano scale flows is less well recognized. Equation (6) only shows how vorticity $\zeta=\operatorname{curl}(\mathbf{v})$ is generated: it gives no indication of how this vorticity evolves into the discrete vortex structures observed experimentally. As noted in the Introduction, there are several factors involved in this process which are related to the geometry of the device. At the same time, the present study is not concerned with wall generated vorticity and possible flow separation.

As noted before, the non-linear term in the linear momentum equation is often neglected in these low velocity micro/nano scale flows. If that is done in equation (6) it follows that the $\operatorname{curl}(\mathbf{f})$ term is collinear with the vorticity vector. In this 


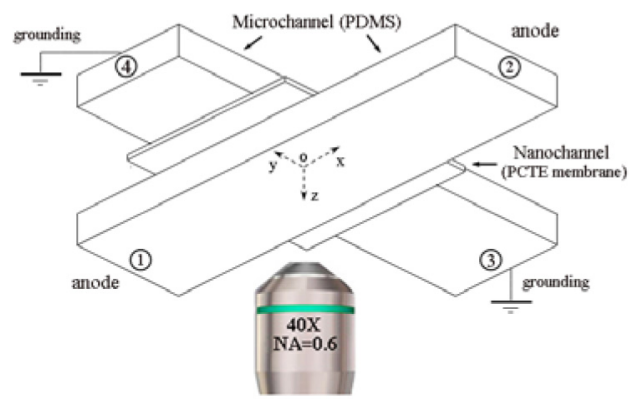

Figure 1: The micro/nano device used in the experiments. Not to scale.

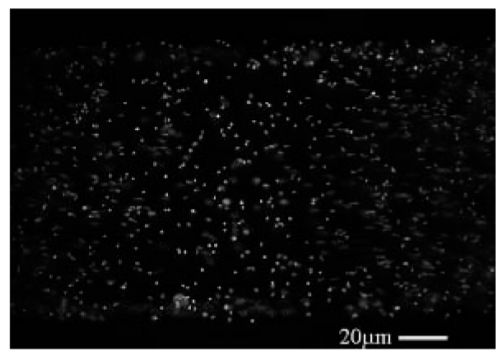

Figure 2a $\left(t^{*}=0.25\right)$

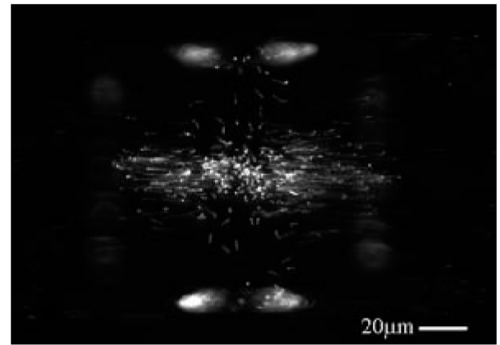

Figure 2c $\left(t^{*}=0.98\right)$

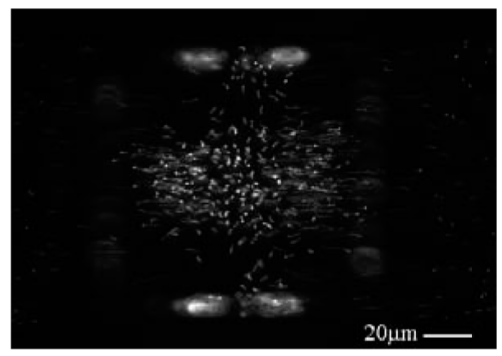

Figure $2 \mathrm{~b}\left(t^{*}=0.65\right)$

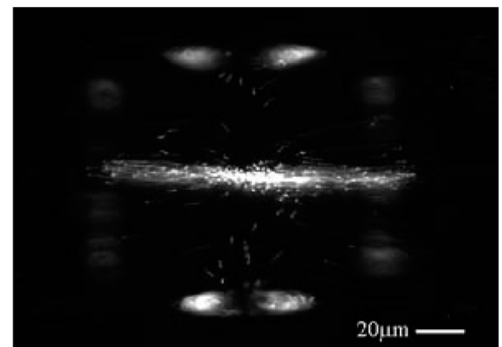

Figure $2 \mathrm{~d}\left(t^{*}=1.28\right)$

Figure 2: Vortex evolution.

case equation (6) specifies the direction of the vorticity field. This directionality of the vorticity field then implies the initial direction of the evolving vortex. The experiments discussed below study the significance of equation (6) for vorticity generation in a specific micro/nano scale flow (see Figure 1 for the device in question and Figure 2 for the emergence of discrete vortices from that vorticity). 


\section{The experimental method}

The experimental study discussed herein formed part of a research program on micro/nano scale fluid mechanics that has been carried out at the Laboratory for Non-Linear Mechanics (LNM) in the Chinese Academy of Science, Beijing, China. In the course of that experimental program it was observed that vortices were generated inside a micro/nano scale device (that shown in Figure 1) as the flow field developed under the action of an applied electric field. All the experiments reported herein relate to a visual study of the flow field in that same device. Preliminary results have been reported in Silber-Li et al. [12] and Yu and Silber-Li [13].

The theoretical study outlined in section 2 above suggests how vorticity can be generated in micro/nano scale flow devices due to the applied electric field interacting with the distribution of ions. The interpretation of the flow field development discussed herein will ignore the viscous flow boundary layers that develop on the walls of the device since the rotational sense of such wall vorticity is not consistent with the observed vortex structures. While the overall flow development is crucially dependent upon the device geometry, only one geometry, that shown in Figure 1, was tested. Flows in other devices, adopting different geometries, have been reported upon in the literature and have found different vortex configurations.

The interest herein concerns the coalescence of the generated vorticity into discrete vortex structures. This process is examined experimentally since a study of the field equations (equations 5a,b,c,d above), in isolation from the boundary conditions, cannot provide much information about this vortex development process. Indeed, a numerical solution of the full set of equations is required for that.

\subsection{Experimental setup}

As noted above, the experimental work reported herein was carried out at the LNM, and the chip adopted for the experiments that was shown in Figure 1 above. In order to allow visualization of the flow field the device was mounted upon an inverted fluorescence microscope (type Olympus $I X-71$ fitted with a $40 \times / 0.6$ lens). The flow field images were recorded by an EMCCD camera (type Andor 885). The electrical potential was obtained from a DC power supply (Zolix Instruments Co., Ltd, Model $D Y Y-12 C$ ) whose output was stable to $\pm 0.03 \% / h$. The voltage range was 0 to $1100 \mathrm{~V}$.

\subsection{The micro-chip}

The hybrid micro/nano-channels used in the experimental study (see Figure 1) were constructed from two polydimethyl-siloxane (PDMS) micro-channels connected together across a polycarbonate nano-porous membrane (PCTE). The micro-channels were $100 \mu m \pm 0.1 \mu m$ in width, $20 \mu m \pm 0.1 \mu m$ in depth and 
$9 \mathrm{~mm} \pm 0.02 \mathrm{~mm}$ in length. The two channels were mounted orthogonally across the membrane as shown in Figure 1. The nano-pores of the PCTE membrane were distributed randomly and had a nominal diameter of $50 \mathrm{~nm}$, with a porosity of less than $1.57 \%$. The membrane thickness was $8 \mu \mathrm{m}$ with a measured error of $\pm 0.8 \mu \mathrm{m}$. The random nature of the porosity demands that the vortex structures observed in the experiment are not directly related to the membrane pore geometry.

\subsection{The working fluid and tracer particles}

The buffer solution concentration adopted in the experiment was $10 \mathrm{mM}$ Borax at $p H 9.2$. The fluorescent particles used to visualize the motion were of $200 \mathrm{~nm}$ diameter with a negative surface charge. The particle density, $1.05 \mathrm{~g} / \mathrm{cm}^{3}$, was close to that of water and any buoyancy effects were very small. These tracer particles were excited by a mercury lamp light source and made visible with a filter wavelength of $616 \mathrm{~nm}$. The particles were diluted into DI water to a relative volume concentration of $5 \times 10^{-5}$ and then diluted into the Borax solution with a $1: 2$ volume ratio.

\subsection{Experimental processes}

The experiment started with the bottom channel of the micro/nano-channel device being filled with the buffer solution containing the fluorescence particles. The upper channel was filled with the pure borax buffer solution. Platinum electrodes were inserted into the reservoirs of the micro-channel to provide the electrical potential across the PCTE membrane. A DC voltage of $2 \mathrm{~V}$, was applied to the reservoirs of the bottom channel while those of the upper channel were grounded (Figure 1). This voltage was too low to induce electrolysis. A detailed discussion of the concentration profile development was reported in Yu and Silber-Li [13]. It is the passage of this concentration profile through the channel that provides the concentration gradient, $\nabla\left(c^{\alpha}\right)$, that featured in equation (6) for vorticity generation.

The focal plane of the EMCCD camera was adjusted to view, from vertically below, flow in the lower micro-channel. The focal plane was located $15 \mu \mathrm{m}$ below the bottom surface of the nano-porous membrane. The maximum image size was $(200 \times 200) \pm 0.26 \mathrm{~mm}$. A sequence of images were recorded with an exposure time of $\Delta t_{p}=100 \mathrm{~ms}$ and an interval time of $\Delta t=130 \mathrm{~ms}$. Images from the experiments showing the evolution of the vortical structures are shown in Figure 2 and will be discussed below.

\section{Results from the experiment}

The initial stages of vortex formation in the device shown in Figure 1 can be observed by flow visualization techniques and the results of such an experiment are shown in Figure 2 using the tracer particles as discussed above. For the flows 


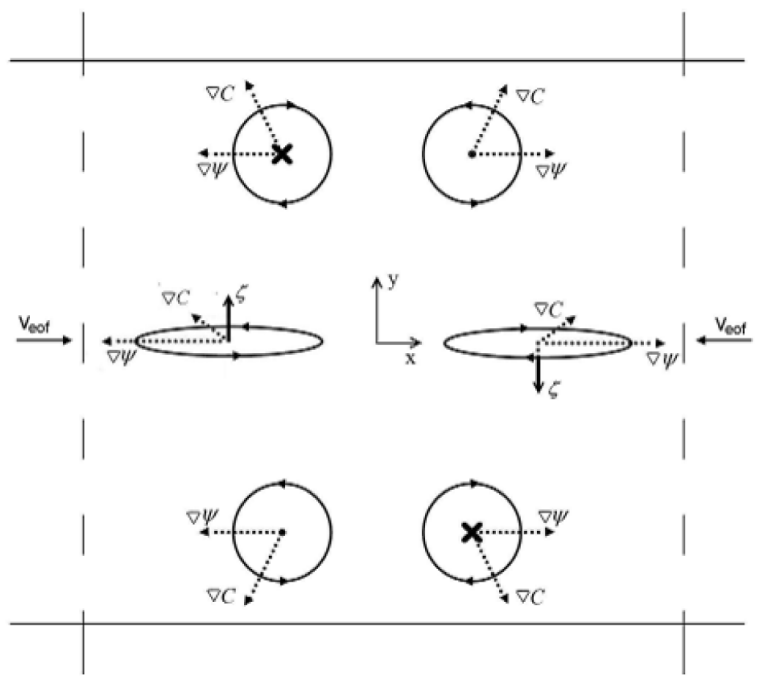

Figure 3: Schematic showing the direction of the eddies relative to the two local gradients, $\nabla\left(c^{\alpha}\right)$, and, $\nabla(\Psi)$ in the junction region of the hybrid macro/nano channel. In this figure, " $\mathbf{x}$ " represents a clockwise rotating vortex (with rotation along the $-z$ axis). At the same time the symbol "." represents count-clockwise rotating vortices (rotation is along the $z$ axis).

considered herein there is no wall vorticity included as the vortices develop in the flow field and not adjacent to solid boundaries.

\subsection{Evolution of the vortices}

At the start of the experiment, the fluorescence particles in the bottom microchannel were at rest as discussed above in section 3. Motion of the particles was, however, observed after the electric field was applied across the channel. It is meaningful to discuss the flow evolution in a non-dimensional time scale, $t^{*}$, where: $t^{*}=v_{\text {eof }} t / \ell$. Here, $\ell$ is the depth of the micro-channel and $v_{e o f}=$ $\epsilon \zeta_{w} E / \mu$ denotes the Smoluchowski velocity. Also $t$ denotes the physical time, measured in seconds, from the instant when the electric field was applied. For the present application the wall zeta potential had the value $\zeta_{w}=-30 \mathrm{mV}$. The electric field was $E \approx 2 \mathrm{~V} / \mathrm{cm}$ while the value for the dialectric constant was $\epsilon=7 \times 10^{-10} c^{2} / \mathrm{Nm}^{2}$. The fluid viscosity was taken to be $\mu=10^{-3}$ Pa.s. Figure 2a shows the state at time $t^{*}=0.25(t=1.25 \mathrm{~s})$. At $t^{*}=0.65(t=3.25 \mathrm{~s})$ three dominant pairs of vortices began to emerge from the cloud of particles (Figure 2b). Two pairs of small vortices are located near the upper and bottom side walls. These vortices were seen to rotate in opposite direction. A pair of vortices (the principle vortices) was found to be located in the (horizontal), $x \sim y$, plane in 

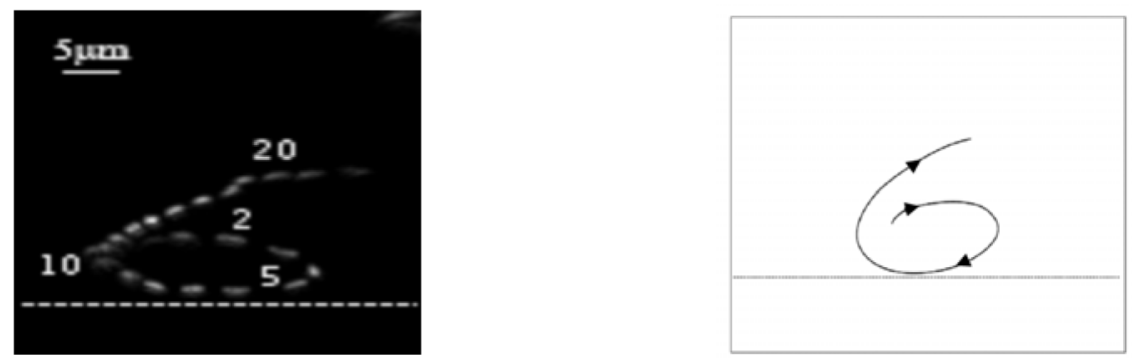

4a: lower-right vortex $\left(2 \mathrm{~V} ; \Delta t_{p}=20 \mathrm{~ms} ; \Delta t=30 \mathrm{~ms}\right.$ with $\left.T=22 \Delta t\right)$
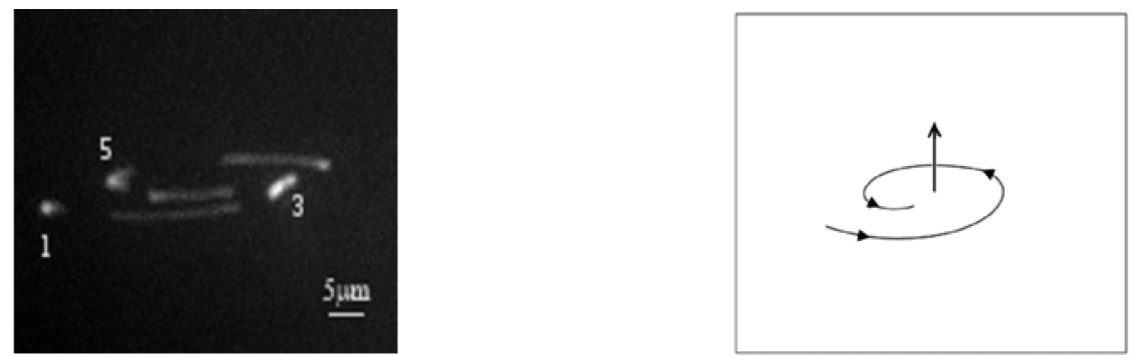

4b: center-left vortex $\left(5 \mathrm{~V} ; \Delta t_{p}=20 \mathrm{~ms} ; \Delta t=40 \mathrm{~ms}\right.$ with $\left.T=6 \Delta t\right)$

Figure 4: Traces for two of the vortices.

the lower micro-channel $15 \mu \mathrm{m}$ from the membrane. At time $t^{*}=0.98(t=4.9 \mathrm{~s})$ the particles in the principle vortices are migrating towards the central plane of the micro-channel as shown in Figure $2 \mathrm{c}$. The last figure, Figure $2 \mathrm{~d}$, shows the location of the vortices at $t^{*}=1.28(t=6.4 s)$. At this time all the particles in the principle vortices are located on the central plane. Figure 3 summarizes the final vortex locations and their sense of rotation.

\subsection{Rotation directions of the vortices}

To illustrate the vortex rotation more clearly, the motion was observed with fewer particles than used for Figure 2. Figure 4 presents a pair of vortex images and their sketch maps. The vortex in Figure $4 \mathrm{a}$ is located at the bottom-right hand corner on Figure 3. The photograph in Figure 4a was obtained by overlapping 22 images with the tracer particle captured at a time $T=22 \Delta t$. The numbers in the picture indicate the time order of the tracer particle as it moved within the vortical flow. For locations 2, 5, 10 the particle was within the vortex boundary. Then for location 10, 20 the particle departs from the vortex and migrates towards the center of the micro-channel. This vortex rotated clockwise and the axis of rotation was essentially along the $-z$ axis. The dotted-line on the figure indicates the wall of the micro-channel. Similarly for the vortex in the lower-left (on Figure 3), the 
vortex rotated counter-clockwise and the axis of rotation was along the $+\mathrm{z}$ axis. The sense of rotation of the upper left and upper right vortices is as shown on Figure 3. For the principle vortices resided in the center of the junction region, it was observed that the tracer particles of vortex in the center-left moved round the vortex as indicated by the numbers on the tracer particles (Figure 4b). However, the rotation axis of this vortex is along $+y$ axis. Symmetrically for the vortex in the center-right, its rotation axis is along $-y$ axis. The vortex system is symmetric about the $y-z$ plane.

\subsection{Velocity field in the vortices}

The tracer particle velocities were computed from the images in Figure 4. For the vortex in Figure $4 \mathrm{a}$, the trace moved $2.5 \mu \mathrm{m}$ during the time interval $\Delta t_{p}$ so that its velocity was $125 \mu \mathrm{m} / \mathrm{s}$. This data implies a Strouhal number $S_{t}=0.021$ for the center vortex and a Reynolds number (based upon $v_{e o f}$ ) of $R_{e}=0.0013$. Hence $R_{e} / S_{t} \approx 0.062$. The creeping flow condition, $R_{e} / S_{t} \ll 1$, is not well satisfied. From Figure $4 \mathrm{~b}$, the velocity in the center left vortex was about $270-500 \mu \mathrm{m} / \mathrm{s}$. As it is noted in section 4.1, the Smoluchowski velocity $v_{e o f}$ is $13 \mu \mathrm{m} / \mathrm{s}$ in $5 \mathrm{~V}$. The electro-phoresis velocity of the fluorescent particle was $22 \mu \mathrm{m} / \mathrm{s}\left(v_{e p}=\epsilon \zeta_{p} E / \mu\right.$, here, $\zeta_{p}=-50 \mathrm{mV}$ denotes the zeta potential of the particle surface). Since the direction of $v_{e o f}$ and $v_{e p}$ are opposite, the net velocity of particle movement is only $10 \mu \mathrm{m} / \mathrm{s}$. Hence the tracer velocity gives the line speed of the vortices.

\section{Discussion of the results}

The present section discusses the distribution of both the electric field gradient, $\nabla(\Psi)$, and the ion concentration gradient, $\nabla\left(c^{\alpha}\right)$, in order to identify the sense of vortex rotation shown in Figure 3 and to compare with the experimental data in Figures 3 and 4.

\subsection{Distribution of $\nabla(\Psi)$}

The electric field strength, $\mathbf{E}=-\nabla(\Psi)$, with potential $\Psi$ was introduced in section 2 above. This potential $\Psi$ includes two parts: that due to the applied electric field and the EDL potential $\zeta_{w}$. For the buffer solution used in the present experiments, the EDL thickness was approximately $10 \mathrm{~nm}$ and the corresponding potential $\zeta_{w} \approx 30 \mathrm{mV}$. Hence the influence of $\zeta_{w}$ on the potential $\Psi$ for the bulk flow in the micro-channel can be neglected. According to Figure 1, the applied voltage is positive in the lower micro-channel and negative in the upper microchannel. Hence the direction of the gradient, $\nabla(\Psi)$, was from the upper to the lower micro-channel (the electric potential is increasing). This implies that in the lower micro-channel the potential gradient, $\nabla(\Psi)$, increased from the center (that is in the junction region) to both ends 1 and 2 (see Figure 1). 


\subsection{Distribution of $\nabla\left(c^{\alpha}\right)$}

The applied electric field produces enrichment in the upper micro-channel and depletion in the lower channel. From previous experimental results (see Yu and Silber-Li [13]), the depletion zone in the lower micro-channel will develop from the junction region and pass along the micro-channel towards the reservoirs. Therefore, the gradient, $\nabla\left(c^{\alpha}\right)$, is directed along the normal to the depletion zone profile and toward the higher concentration.

\subsection{Vortices with $\nabla\left(c^{\alpha}\right)$ and $\nabla(\Psi)$}

The vortices rotate about the span of the vector $\nabla\left(c^{\alpha}\right) \times \nabla(\Psi)$. Now consider each vortex shown in Figure 3. For the upper-left vortex as noted in Figure 3a, $\nabla(\Psi)$ and $\nabla\left(c^{\alpha}\right)$ are in the $x-y$ plane while $\nabla(\Psi)$ is along the $-x$ axis and $\nabla\left(c^{\alpha}\right)$ is along the normal direction of the depletion zone. While the front of the depletion zone moves away from the center of the micro-channel, it does so most rapidly in the region near the nano-porous membrane. That is, the normal to the front is, locally, not along the $-x$ axis but rather between the $-x$ and the $y$ directions (see Figure 3). Therefore, rotation of upper-left vortex is along the $-z$ axis. Similarly, the rotation of upper-right vortex is along the axis $z$. By means of a similar analysis, the rotation of the lower-left vortex is along axis $\mathrm{z}$ and the rotation of lower-right vortex is along axis $-z$. For the principle vortices at centerleft, the $\nabla(\Psi)$ and $\nabla\left(c^{\alpha}\right)$ are both in the $x-z$ plane, with $\nabla(\Psi)$ along the $-x$ axis and $\nabla\left(c^{\alpha}\right)$ along the normal direction to the depletion zone. Therefore, rotation of the center-left vortex is along the $y$ axis. Similarly, the rotation of the center-right vortex was along the $-y$ axis.

\section{Conclusions}

Vortices in a three-dimensional hybrid micro/nano channel, subjected to an applied electric field, have been visualized. In addition, an analysis of the vorticity origin, based on the Navier Stokes and Nernst-Planck equations, was undertaken. The main points from the study are as follows:

(1) Three pairs of vortices were found from the experimental images. The rotations of the vortices near the sidewalls are orthogonal to the principle vortices in center channel. This differs from the situation in two-dimensional channels where the axes of rotation of the vortices are parallel. The velocities of the vortices was also calculated. The results show that the line velocities are much larger than the electro-osmotic flow velocity, and the directions of the velocity near the wall were the same as $v_{e o f}$, but in the channel center, the velocity was opposite to this.

(2) The theoretical analysis has indicated that vorticity in the micro-channel resulted from the cross product of the concentration gradient and the electric field gradient. It was found that the experimentally observed vortices were consistent with this finding. 
The flow visualization experiments reported herein illuminated the complexity of the flow field resulting from the applied electric field interacting with charged ions in the buffer solution. There is a need for further work.

\section{Acknowledgements}

The authors wish to thank the following organizations for financial support: Knowledge Innovation Project of The Chinese Academy of Sciences (KJCX2YW-H18) and the Natural Science Foundation of China (10872203).

\section{References}

[1] Takhistov, P.; Duginova, K. and Chang, H. C: Electrokinetic Mixing Vortices due to Electrolyte Depletion at Micro-channel Junctions. J. Colloid and Interface Sci., vol. 263, pp 133-143, 2003.

[2] Park, S. Y; Russo, C. J; Banton, D. and Stone, H. A: Eddies in a Bottleneck: An Arbitrary Debye Length Theory for Capillary Electro-osmosis. J. Colloid and Interface Sci., vol. 297, pp 832-839, 2006.

[3] Kim, S-J.; Wang, Y-C.; Lee, J-H.; Jang, H. and Han, J: Concentration Polarization and Nonlinear Electro-kinetic Flow Near a Nonfluidic Channel. Phys. Rev. Lett., 99:044501, 2007.

[4] Huang, K-D and Yang R-J: Formation of Ionic Depletion/enrichment zones in a Hybrid Micro/nanochannel. Microfluid Nanofluid, vol. 5, pp 631-638, 2008.

[5] Jin, X.; Joseph, S.; Bohn, P. W. and Aluru, N. R: Induced Electrokinetic Transport in Micro-Nanofluidic interconnect. Langmuir, vol. 23, pp 1320913222, 2007.

[6] Moffatt, H. K: Viscous and Resistive Eddies near a Sharp Corner. JFM, vol. $18,1964$.

[7] Gurtin, M. E: Introduction to Continuum Mechanics. Academic Press, New York, 1981.

[8] Stokes, G. G: On the Theories of the Internal Friction of Fluids in Motion and of the Equilibrium and Motion of Elastic Solids. Trans. Camb. Phil. Soc. vol. 8, pp 287-319, 1845.

[9] Stakgold, I: Boundary Value Problems of Mathematical Physics. Macmillan, 1967.

[10] Silber-Li, Z-H, Kong, G-P, Zheng, X, Yu, Q and Moulden, T. H: "Vorticity and Pressure Fields in Micro/Nano Channel Flows". In preparation.

[11] Silber-Li, ZH; Kong, GP; Yu,Q and Moulden, T. H: Observation of Vorticity generation in Micro/nano-channel Flows Subject to an Electric Field. Proc. 11th Asian Symp. Visualization, Japan, 2011.

[12] Silber-Li, ZH; Yu, Q and Kong, GP: The Experiments about Ionic ExclusionEnrichment Effects in a Hybrid Micro/Nano-channel. Proc. 2nd Euro. Conf. on Microfluidics, France, 2010.

[13] Yu, Q and Silber-Li, ZH: Measurement of the Ion-depletion Zone Evolution in a Micro/Nano-channel MicroFluid-Nanofluid, vol. 11, pp 623-631, 2011. 